\title{
The Crossover between Liquid and Solid Electron Phases in Quantum Dots: A Large-Scale Configuration-Interaction Study
}

\author{
Massimo Rontani ${ }^{\mathrm{a}, *}$ Carlo Cavazzoni ${ }^{\mathrm{a}, \mathrm{b}}$ Guido Goldoni ${ }^{\mathrm{a}, \mathrm{c}}$ \\ a INFM National Research Center S3, Modena, Italy \\ ${ }^{\mathrm{b}}$ CINECA, Casalecchio di Reno, Italy \\ ${ }^{\mathrm{c}}$ Dipartimento di Fisica, Università degli Studi di Modena e Reggio Emilia, Modena, Italy
}

\begin{abstract}
We study the crossover between liquid and solid electron phases in a two-dimensional harmonic trap as the density is progressively diluted. We infer the formation of geometrically ordered phases from charge distributions and pair correlation functions obtained via a large scale configuration interaction calculation.
\end{abstract}

Key words: quantum dots, configuration interaction, Wigner crystal PACS: 73.21.La, 73.20.Qt

\section{Introduction}

Semiconductor quantum dots [1,2,3] (QDs) are nanometer sized region of space where free carriers are confined by electrostatic fields. Almost all QD-based applications rely on electronic correlation effects which are prominent in these systems. The dominance of interaction in QDs is evident from the multitude of strongly correlated few-electron states measured or predicted under different regimes: Fermi liquid, "Wigner molecule" (the precursor of Wigner crystal in two-dimensional bulk), charge and spin density wave, incompressible state reminescent of fractional quantum Hall effect in two dimensions [2].

The few-body problem has been faced mainly via Hartree-Fock method, Density Functional Theory, Configuration Interaction (CI), and Quantum Monte Carlo

\footnotetext{
* Corresponding Author:

Email address: rontani@unimore. it (Massimo Rontani).
} 
(QMC) [1,2]. Contrary to mean-field methods, CI and QMC methods allow for the treatment of Coulomb correlation with arbitrary numerical precision, and, therefore, represent the natural choice for strongly correlated regimes. In addition, CI is a straightforward approach with respect to QMC, and gives access to both ground and excited states at the same time, its main limitation being its computational cost. Here we apply the CI method to the the strongly correlated regime par excellence, namely the crossover region between Fermi liquid and Wigner crystal [2]. We find evidence of the formation of Wigner molecules, and confirm previous QMC calculations $[4,5]$.

\section{The crossover between liquid and solid phases}

We consider a few electrons in a two-dimensional harmonic trap, which was proven to be an excellent model for different experimental setups [2]. The QD effectivemass Hamiltonian is:

$$
H=\sum_{i}^{N}\left[-\frac{\hbar^{2}}{2 m^{*}} \nabla^{2}+\frac{1}{2} m^{*} \omega_{0}^{2} \rho_{i}^{2}\right]+\frac{1}{2} \sum_{i \neq j} \frac{e^{2}}{\kappa\left|\boldsymbol{\rho}_{i}-\boldsymbol{\rho}_{j}\right|} .
$$

Here $m^{*}$ and $\kappa$ are the effective mass and the static relative dielectric constant of the host semiconductor, respectively, $\boldsymbol{\rho}_{i} \equiv\left(x_{i}, y_{i}\right)$ is the position of the $i$-th electron, $\omega_{0}$ is the characteristic frequency of the trap. The cylindrical spatial symmetry group of the system is $D_{\infty h}$, and the single-particle QD wavefunctions, the so called Fock-Darwin (FD) orbitals [1], have an azimuthal and a radial quantum number, respectively. The good global quantum numbers of the few-body system are the total angular momentum in the direction perpendicular to the plane, $M$, the total spin, $S$, and its projection along the $z$-axis, $S_{z}$.

We solve numerically the few-body problem of Eq. (1), for the ground state at different numbers of electrons, $N$, by means of the CI method [6]: we expand the many-body wavefunction in a series of Slater determinants built by filling in the FD orbitals with $N$ electrons, and consistently with global symmetry constraints. On this basis, the Hamiltonian (1) is a large, sparse matrix that we diagonalize by means of a Lanczos parallel routine [7]. Note that, before diagonalization, we are able to build separate sectors of the Fock space corresponding to different values of $\left(M, S, S_{z}\right)$. To this aim, we rely on tabulated Clebsh-Gordon coefficients obtained, once for all, via numerical diagonalization of the $S^{2}$ matrix (cf. Sec. 2.3 of Ref. [8]). We use, as a single-particle basis, up to 36 FD orbitals, and we need to diagonalize matrices of linear dimensions up to $\approx 10^{6}$.

The way of tuning the strength of Coulomb correlation in QDs is to dilute electron density. At low enough densities, electrons pass from a "liquid" phase, where 


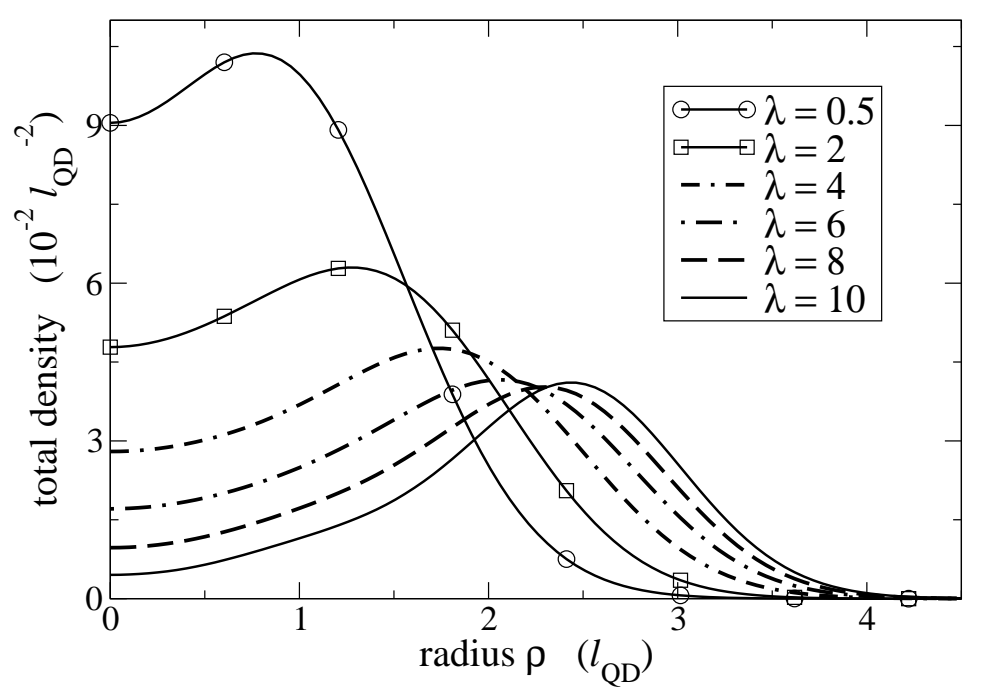

Fig. 1. Normalized total density of the five-electron ground state as a function of the radius, $\rho$, for different values of the correlation strength parameter, $\lambda$. The length unit is the characteristic dot radius, $\ell_{\mathrm{QD}}$.

low-energy motion is equally controlled by kinetic and Coulomb energy, to a "crystallized" phase, reminescent of the Wigner crystal in the bulk, where electrons are localized in space and arrange themselves in a geometrically ordered configuration such that electrostatic repulsion is minimized [2,3]. The typical QD lateral extension is given by the characteristic dot radius $\ell_{\mathrm{QD}}=\left(\hbar / m^{*} \omega_{0}\right)^{1 / 2}, \ell_{\mathrm{QD}}$ being the mean square root of $\rho$ on the FD lowest-energy level. As we keep $N$ fixed and increase $\ell_{\mathrm{QD}}$, the Coulomb-to-kinetic energy ratio $\lambda=\ell_{\mathrm{QD}} / a_{\mathrm{B}}^{*}\left[a_{\mathrm{B}}^{*}=\hbar^{2} \kappa /\left(m^{*} e^{2}\right)\right.$ is the effective Bohr radius of the dot] [4] increases as well, driving the system into the Wigner regime.

Figure 1 shows the total electron density vs. $\rho$, for $N=5$ and for different values of $\lambda$. The density is normalized to $N$, and the ground state remains $\left(M, S, S_{z}\right)=$ $(1,1 / 2,1 / 2)$ across the liquid-solid transition. While in the non-interacting case $(\lambda \rightarrow 0)$ the charge is homogeneously spread across the dot, approximately between $\rho=0$ and $\rho=2 \ell_{\mathrm{QD}}$, for $\lambda>4$ an outer ring develops while the weight in the inner region is stronlgy depleted. For $\lambda>8$ a well resolved peak forms at $\rho \approx 2.5 \ell_{\mathrm{QD}}$, corresponding to the "freezing" of the electron liquid into a Wigner molecule made of five electrons sitting at the corners of a regular pentagon. This interpretation is supported by our analysis of the pair correlation function [3] and by the excellent agreement between the computed values of ground-state energies of our calculation and those obtained via QMC [4,5].

The "crystallization" of the five-electron molecule is confirmed by the analysis of the spin-resolved density, normalized to the number of electrons with a given spin [Fig. 2]. In the liquid phase [left panel of Fig. 2, $\lambda=2$ ], since the number of electron is odd, densities for the two spin species of course differ. In the Wigner limit (right panel, $\lambda=10$ ), instead, the system becomes classical and the spin 

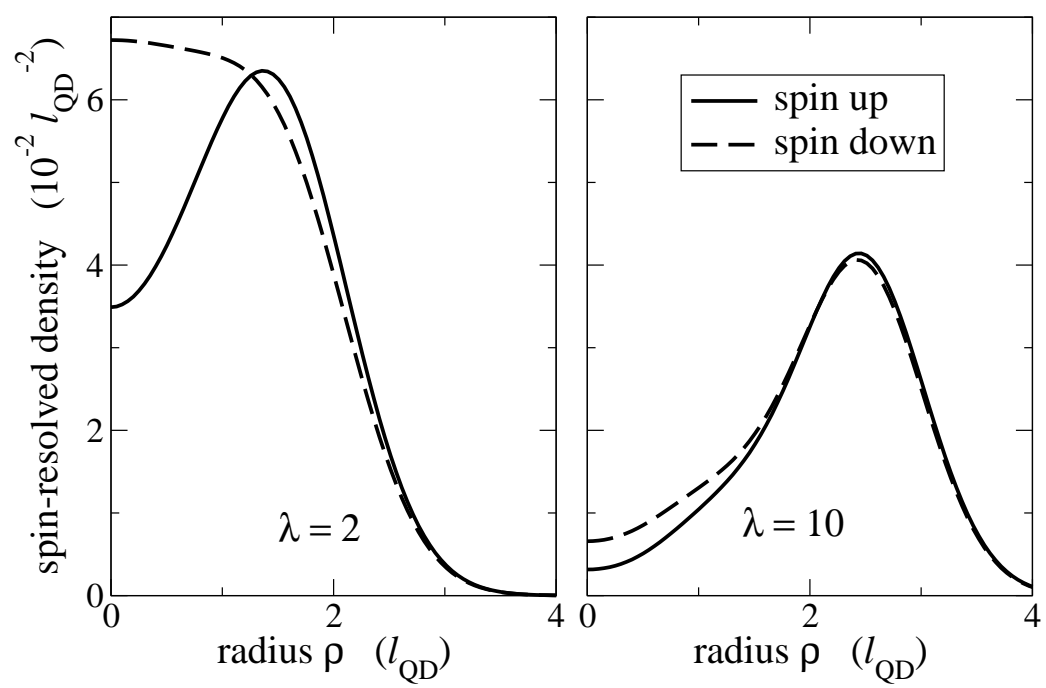

Fig. 2. Normalized spin-resolved density of the five-electron ground state as a function of the radius, $\rho$. Left panel: $\lambda=2$. Right panel: $\lambda=10$. The length unit is the characteristic dot radius, $\ell_{\mathrm{QD}}$.

degree of freedom irrelevant: densites for electrons with spin up or down coincide.

This paper is supported by MIUR-FIRB RBAU01ZEML, miur-cofin 2003020984, Iniziativa Trasversale INFM Calcolo Parallelo 2004, MAE-DGPCC.

\section{References}

[1] L. Jacak, P. Hawrylak, A. Wójs, Quantum dots, Springer, Berlin, 1998.

[2] S. M. Reimann, M. Manninen, Electronic structure of quantum dots, Rev. Mod. Phys. 74 (2002) 1283.

[3] M. Rontani, G. Goldoni, E. Molinari, Few-electron liquid and solid phases in artificial molecules at high magnetic field, in: R. Fazio, V. F. Gantmakher, Y. Imry (Eds.), New directions in mesoscopic physics, Kluwer, Dordrecht, 2003, p. 361.

[4] R. Egger, W. Häusler, C. H. Mak, H. Grabert, Crossover from fermi liquid to wigner molecule behavior in quantum dots, Phys. Rev. Lett. 82 (1999) 3320-3323.

[5] B. Reusch, R. Egger, Impurity effects in few-electron quantum dots: Incipient wigner molecule regime, Europhys. Lett. 64 (2003) 84-90.

[6] M. Rontani, S. Amaha, K. Muraki, F. Manghi, E. Molinari, S. Tarucha, D. G. Austing, Molecular phases in coupled quantum dots, Phys. Rev. B 69 (2004) 85327.

[7] R. B. Lehoucq, K. Maschhoff, D. C. Sorensen, C. Yang, ARPACK computer code, available at http://www. caam.rice.edu/software/ARPACK/.

[8] R. Pauncz, Spin eigenfunctions - Construction and use, Plenum Press, New York, 1979. 